\title{
LES CONTRAINTES D'UN PARADOXE. LES LOIS CONSTITUTIONNELLES INCONSTITUTIONNELLES : RÉFLEXIONS À PARTIR DU CAS INDIEN
}

\author{
ARNAUD LE PILLOUER
}

Commençons par devancer une objection possible - relative à la consistance même du paradoxe en question. À l'évidence, il tient d'abord au vocabulaire employé, et de bons auteurs soutiendraient avec quelque apparence de raison qu'il suffit de remplacer «lois constitutionnelles » par «lois de révision » pour que le paradoxe s'évanouisse instantanément.

Il nous semble pourtant que ce dernier serait alors moins surmonté que repoussé. En effet, cette substitution terminologique repose sur l'idée que le « pouvoir de révision » est un pouvoir constitué, qui se distingue donc par nature du (véritable) pouvoir constituant. Il demeure cependant que ledit pouvoir de révision crée bien des règles constitutionnelles, puisque les lois qu'il adopte sont incorporées au texte de la constitution et y figurent au même titre que les dispositions initiales : en d'autres termes, le pouvoir de révision est bien, au moins quant à son objet, un pouvoir constituant. Non seulement, donc, le paradoxe initial ne s'efface pas complètement (il y a bien des « lois constitutionnelles inconstitutionnelles »), mais on voit en apparaître un autre, puisque le pouvoir de révision apparaît comme étant à la fois constituant et constitué - antinomie que l'on s'efforce de dissimuler par l'emploi de l'obscure expression de «pouvoir constituant dérivé».

Mais si le paradoxe persiste, c'est peut-être tout simplement qu'il est inévitable : il est en effet le simple résultat de l'existence, dans la constitution, d'une procédure spéciale de révision constitutionnelle. Inclure en effet (pour des raisons d'ordre politique évidentes, sur lesquelles il est inutile de s'appesantir) un tel mécanisme dans la constitution, c'est assigner à cette dernière une tâche éminemment paradoxale - celle de déterminer les conditions de sa propre création. Sans discuter ici la teneur ni les implications d'une telle ambition, rappelons simplement que, poussée à son terme (en l'occurrence lorsque l'on prétend utiliser cette procédure de révision pour modifier la disposition instituant cette même procédure), elle aboutit à une proposition auto-référentielle, c'est-à-dire à une absurdité logique - ainsi qu'Alf Ross l'a fort bien mis en évidence'1.

Mais souligner qu'il y a bien quelque chose de paradoxal dans l'idée que des lois constitutionnelles puissent être déclarées inconstitutionnelles ne signifie pas

1. AlfRoss : «À propos del'auto-référence et d'uneénigme du droit constitutionnel », in Introduction à l'empirisme juridique, trad. par E. Millard et E. Matzner, Paris, LGDJ, 2004, p. 205 et $\mathrm{s}$. 
qu'elle doive être pour cette raison abandonnée. Le droit en effet s'accommode fort bien des paradoxes et - quoi qu'on en dise - des absurdités logiques ; aussi n'y a-t-il aucune raison de rejeter le contrôle des lois de révision pour ce seul motif ${ }^{1}$.Tel ne sera donc pas notre propos.

L'intérêt de cette question est même patent, pour au moins deux raisons. D'un point de vue très général, on doit tout d'abord observer que les cours constitutionnelles acceptant de se prononcer sur la validité d'amendements constitutionnels tendent à se multiplier. Si l'on laisse de côté le cas très particulier de l'Afrique du Sud, où la Cour constitutionnelle a été littéralement intégrée au processus constituant originaire ${ }^{2}$, les exemples les plus classiques sont à trouver parmi les plus grandes cours européennes - notamment en Allemagne $^{3}$ et en Italie ${ }^{4}$, sans compter la plus ancienne d'entre elles, la Cour constitutionnelle autrichienne (qui statue cependant dans un contexte juridique un peu différent ${ }^{5}$ ). Très récemment encore, des cours constitutionnelles ont, à l'étranger, franchi le pas, comme en Turquie 6 , ou au Bénin ${ }^{7}$ par exemple. Quant à la France, si certains ont pu croire que la décision du 26 mars 2003 mettrait fin au débat, ils ont depuis été fermement démentis par les faits, tant se sont

1. Pour reprendre la formule définitive de Peter Suber, de même que « la loi humaine peut être immorale sans cesser d'être la loi, il semble qu'elle puisse être illogique sans cesser d'être la loi » (P. Suber, The Paradox of Self-Amendment: A Study of Logic, Law, Omnipotence, and Change, New York, Peter Lang, 1990).

2. On peut, sur le sujet, se reporter à Xavier Philippe : « Le contrôle des lois constitutionnelles en Afrique du Sud », Cahiers du Conseil constitutionnel, n 27-2009, p. 22.

3. La Cour constitutionnelle fédérale allemande a en effet estimé, à partir d'une décision du 15 décembre 1970 Écoutes téléphoniques, qu'il lui appartenait de vérifier qu'une révision de la constitution ne viole pas les dispositions de l'art. 79, al. 3 de la Loi fondamentale.

4. La Cour constitutionnelle italienne a estimé, dans sa décision du 29 décembre 1988 (arrêt n 1146/1988), que la Constitution italienne contenait « certains principes suprêmes qui ne peuvent être ni violés ni modifiés dans leur contenu essentiel, ni par des lois de révision constitutionnelle, ni par d'autres lois constitutionnelles », cette dernière distinction étant purement formelle (et fondée sur l'objet des amendements).

5. En effet, la Constitution autrichienne distingue deux procédures de révision de la Constitution : pour une révision totale, un référendum obligatoire ponctue la phase parlementaire (qui oblige à une adoption par la ou les chambres à la majorité qualifiée), tandis que lorsque la révision n'est que partielle, le référendum, facultatif, doit être demandé par un tiers des membres de l'une ou l'autre des chambres (Constitution de l'Autriche, art. 44 , § 3). La Cour s'est donc reconnu la fonction de distinguer les révisions partielles des révisions totales - toutes deux étant permises par la Constitution.

6. Par un arrêt du 6 juin 2008, la Cour constitutionnelle turque a déclaré inconstitutionnelle la révision constitutionnelle du 9 février 2008 (cf. par ex. Éric Sales : « La protection du principe constitutionnel de laïcité par la Cour constitutionnelle turque », Revue du droit public, $\mathrm{n}^{\circ}$ 6-2009, p. 1649 et s.).

7. La Cour constitutionnelle béninoise a annulé, par un arrêt du 8 juillet 2006, la loi constitutionnelle du 23 juin 2006. Pour une analyse de l'arrêt, on peut se reporter à : Babakane D. Coulibaley : «La neutralisation du Parlement constituant (à propos de la décision nDCC 06-074 du 8 juillet 2006 de la Cour constitutionnelle du Bénin) », Revue du droit public, $\mathrm{n}^{\circ}$ 5-2009, p. 1493 et $\mathrm{s}$. 
multipliés, depuis cette date, les articles de doctrine prenant position en faveur du contrôle de constitutionnalité des lois de révision. La tentation est même grande de voir dans ces diverses évolutions une sorte de fatalité historique : de même que le contrôle de constitutionnalité des lois ordinaires a fini, malgré les résistances que l'on sait, par être consacré (presque) partout dans le monde, le temps ne serait plus très éloigné où les lois de révision seraient soumises au mêmerégime.

L'intérêt de la question est renforcé, d'un point de vue plus théorique, par la place qu'elle occupe dans la théorie des contraintes juridiques développée par Michel Troper et Véronique Champeil-Desplats. Celle-ci a reçu ses premières formulations dans certains articles de Michel Troper relatifs précisément aux pouvoirs des cours constitutionnelles. Dans l'un d'entre eux notamment, ayant défendu la thèse de leur absolue liberté interprétative, il est amené à préciser que cette liberté n'est évidemment que juridique, et ne saurait être comprise comme une absence de déterminations causales - ce qui explique que les cours fassent un usage apparemment si modéré de cette liberté. Il ajoute que, même si nombre de ces déterminations n'ont rien à voir avec le droit et ne peuvent être découvertes que par les sciences humaines ou sociales adéquates (se rangeant ainsi à une interprétation «pure et stricte » des contraintes juridiques ${ }^{1}$ ), il demeure qu'un certain nombre d'entre elles résulte du «système juridique »- et doit donc être analysé par la science juridique. Or, au premier rang de ces contraintes, figure la présence d'autres autorités (notamment le Parlement) disposant des moyens juridiques de s'opposer aux éventuelles ambitions débridées de juges constitutionnels peu scrupuleux ; et parmi ces moyens juridiques, l'un des plus fréquemment cités par Michel Troper est évidemment la faculté, offerte aux autorités politiques, de surmonter une décision de la cour constitutionnelle par une révision de la constitution. De sorte que, si la cour se reconnaissait le droit de contrôler aussi les lois constitutionnelles, une telle arme serait réduite à néant ${ }^{2}$. En d'autres termes, la constitution au sens matériel n'étant, selon Michel Troper, qu'un système de rapports de force entre des organes déterminant concurremment l'étendue de leurs compétences respectives ${ }^{3}$, la cour prendrait un avantage très conséquent dans ce système en se reconnaissant le droit d'annuler des lois de révision. On comprend dès lors que la question de l'existence ou non d'un contrôle de constitutionnalité des lois de révision soit relativement centrale, si l'on cherche, en s'appuyant sur la théorie des contraintes juridiques, à rendre

1. Pour reprendre la jolie expression utilisée par Mikhaïl Xifaras dans une brillante communication lors de ces journées dont il a malheureusement préféré ne pas publier le texte.

2. Elle ne serait du reste pas la seule, puisque la plupart des moyens de rétorsion dont disposent ces autorités (modification du statut ou du nombre des juges, des compétences de la cour, etc.) nécessitent une révision de la constitution.

3. Michel Troper : «Le problème de l'interprétation et la théorie de la supralégalité constitutionnelle », in Pour une théorie juridique de l'État, Paris, PUF, “Léviathan”, 1994, partic. p. 307 et s. 
compte de la place occupée par les cours constitutionnelles dans nos systèmes juridiques.

Restent à examiner les raisons pour lesquelles on a, dans cette étude, choisi d'aborder cette question, pour le moins générale, à partir du cas indien. Pour celui qui chercherait une réponse dans le texte de la Constitution indienne, ce choix pourrait même paraître surprenant, puisque l'article 368, qui régit la procédure d'amendement à la Constitution, dispose que : «Nonobstant tout autre élément de la Constitution, le Parlement peut, dans l'exercice de son pouvoir constituant amender, que ce soit par voie d'addition, de modification ou de suppression, n'importe quelle disposition dela présente Constitution, en vertu de la procédure prévue dans le présent article» (al.1) ; que : «Rien dans l'article $13^{1}$ ne saurait s'appliquer à un amendement adopté en vertu du présent article » (al. 3); que : « Nul amendement à la présente Constitution (y compris ceux relatifs à la $\mathrm{III}^{\mathrm{e}} \mathrm{Partie}^{2}$ ), adopté ou supposé avoir été adopté en vertu du présent article, ne peut se voir contester devant aucune cour, sur aucun fondement » (al.5). Et l'alinéa 6 de conclure : « Pour ôter tout doute à ce sujet, il est ici rappelé qu'il ne saurait exister aucune limite d'aucune sorte au pouvoir constituant du Parlement d'amender, que ce soit par voie d'addition, de modification ou de suppression, n'importe quelle disposition de la présente Constitution, en vertu de la procédure prévue par le présent article ».

Si l'on en croit le texte de la Constitution indienne, le problème ne se pose donc pas en Inde, puisqu'il interdit de façon plus qu'explicite - redondante - tout contrôle de constitutionnalité des lois constitutionnelles. Mais précisément, si la fréquentation de cette noble discipline qu'est le droit constitutionnel enseigne quelque chose, c'est bien qu'il ne faut pas toujours croire ce que racontent les textes constitutionnels...

De fait, ces interdictions si fermes et si répétitives sont précisément le signe, plus encore le résultat du contrôle que la Cour suprême indienne effectue sur les amendements à la Constitution. Il convient de préciser que la Cour statue par voie d'exception, dans un système de contrôle décentralisé - ce qui, on le verra, explique que ce contrôle existe en dépit desdites dispositions constitutionnelles, et vice versa. En réalité, il s'agit même de la jurisprudence la plus ancienne en ce sens, et du contrôle le plus profond qui soit, ce qui justifie que l'on s'y arrête - ne serait-ce qu'en raison des enseignements que l'on pourrait en tirer pour nos propres systèmes juridiques.

Et s'il s'agit de procéder à une analyse en termes de contraintes, on peut sans doute en distinguer deux sortes : celles qui conduisent les cours à décider de contrôler (ou non) les lois constitutionnelles d'une part, et celles qui pèsent sur l'argumentation que les cours doivent fournir pour justifier ce contrôle, lorsqu'elles décident d'y procéder, d'autre part. En d'autres termes, on analysera les contraintes comme explication du contrôle (1), avant d'examiner les contraintes pesant sur la justification du contrôle (2).

1. Il s'agit d'un article qui garantit les droits de l'homme contre les atteintes que pourrait leur porter la loi.

2. Il s'agit de la Partie relative à la garantie des droits de l'homme. 


\section{LES CONTRAINTES COMME EXPLICATION DU CONTRôLE \\ DES LOIS CONSTITUTIONNELLES}

L'avènement du contrôle de constitutionnalité des amendements constitutionnels par la Cour suprême indienne doit avant tout se comprendre comme le résultat d'un conflit d'ordre politique très fort qui a opposé, entre les années cinquante et la fin des années soixante-dix, le Parlement à la Cour. D'un côté en effet, le Parlement et son gouvernement, dominés par le Parti du Congrès, souhaitaient mettre en œuvre un programme de réformes agraires et de mesures sociales et économiques inspirées par une volonté très affirmée de redistribution des richesses ; de l'autre, la Cour, composée de juges en majorité plus libéraux et plus conservateurs, était portée à s'opposer à la mise en œuvre de cette politique, en s'appuyant, quant à elle, sur la $\mathrm{III}^{\mathrm{e}}$ Partie de la Constitution relative aux droits fondamentaux ${ }^{1}$.

L'aspect le plus intéressant de ce conflit (du moins du point de vue qui sera le nôtre ici) réside dans les évolutions qu'il a connues depuis l'entrée en vigueur de la Constitution, en 1950. Il s'agira donc d'en présenter les différentes phases, correspondant chacune à une prise de position différente de la part de la Cour suprême - et qu'il conviendra d'analyser en termes de « contraintes ».

\section{Première phase : Le rejet du contrôle}

Il n'aura pas fallu attendre longtemps après l'adoption de la Constitution de l'Inde, pour voir la Cour suprême être saisie de cette difficile question. Pourtant, dans cette affaire Sankari Prasad v. Union of India de 1951, la Cour adopte une position mesurée, mais classique ${ }^{2}$ : elle décide que l'article 368 de la Constitution permet d'amender la Constitution, y compris (puisque telle était la question) les droits fondamentaux garantis dans la III ${ }^{\mathrm{e}}$ Partie de la Constitution. Elle ajoute que l'article 13, qui dispose que « l'État ne doit faire aucune loi qui écarte ou limite les droits consacrés dans la présente Partie et (que) toute loi adoptée en violation de cette clause doit, dans la mesure de cette violation, être considérée comme nulle», ne vise pas les amendements à la Constitution mais les seules lois ordinaires ${ }^{3}$.Commebeaucoup de cours constitutionnelles aujourd'huiencore (en

1. Edward McWhinney, Supreme courts and judicial law-making : constitutional tribunals and constitutional review, Dordrecht, Martinus Nijhoff Pub., 1986, p. 134.

2. Sankari Prasad v. Union of India, All India Reporter (A.I.R.) 1951 SC 458.

3. Il s'agissait, dans cette affaire, de statuer sur l'avenir des actions introduites par de grands propriétaires terriens contre des lois qui, adoptées avant la mise en vigueur de la Constitution par l'Union indienne et certains États, avaient été constitutionnalisées par l'Assemblée constituante elle-même dans un $1^{\mathrm{er}}$ amendement à la Constitution (la $9^{\mathrm{e}}$ liste annexée à la Constitution comprend 12 textes de lois, comportant réformes agraires et nationalisations). En statuant en ce sens, la Cour suprême a mis ces lois à l'abri des actions intentées par ces propriétaires. Sur ce point, on peut se reporter à Jean-Louis Halpérin : 
France, aux États-Unis, ou en Irlande, par exemple), la Cour suprême indienne reconnait donc dans cette décision de 1951 la souveraineté du pouvoir de révision, et refuse de connaître des amendements constitutionnels. Du point de vue de la théorie des contraintes (qui applique une grille de lecture «stratégique » aux comportements des acteurs juridiques), ce type de position peut a priori paraître difficile à expliquer : comment comprendre en effet, qu'un acteur renonce à s'octroyer un avantage aussi décisif sur les autres autorités?

Deux explications peuvent sans doute être avancées. La première tient à la forme dans laquelle sont adoptées, dans la plupart des systèmes, les lois de révision. Les constitutions organisent pour la modification de leurs propres dispositions des procédures qui présentent une difficulté variable, mais qui ont en commun de ne permettre ce type de réformes qu'au prix d'un certain consensus institutionnel et/ou politique. En effet, comme il s'agit de toucher aux « règles du jeu » institutionnel, les constituants ont en général le souci d'associer plusieurs organes constitutionnels aux révisions, mais aussi d'obtenir une adhésion aussi large que possible des différentes forces politiques - comme l'atteste l'exigence très fréquente d'une majorité qualifiée pour l'adoption de telles lois. Il faut ajouter que de nombreux textes prévoient la ratification de ces dernières, soit par le peuple, soit, dans les États fédéraux, par les États membres - et parfois même par les deux. On comprend dès lors que les lois de révision soient dotées d'une légitimité si forte que les juges constitutionnels hésitent à les contrôler : dans le cas contraire en effet, la cour risquerait d'apparaître comme un obstacle à la réalisation d'une réforme dont l'adoption par les autorités compétentes témoigne précisément (eu égard à la difficulté de la procédure) de son caractère très consensuel.

Mais la prudence des juges s'explique également, et peut-être surtout, par le fait qu'en procédant à un tel contrôle, ils risqueraient de mettre à mal l'une des justifications les plus puissantes de leur propre pouvoir.Traditionnellement, en effet, dans nos démocraties, la légitimité de la cour constitutionnelle tient à ce qu'elle n'apparaît pas comme un opposant politique à la majorité issue des élections, mais comme une sorte de tiers impartial. Or ce rôle n'est crédible que si son opposition aux réformes souhaitées par ladite majorité se présente comme étant purement technique - et en tout cas non idéologique. C'est sans doute ce qui explique le succès de théories justificatives comme celle dite « de l'aiguilleur », selon laquelle le juge constitutionnel se contenterait d'indiquer la voie à suivre (législative ou constitutionnelle) pour opérer telle ou telle réforme, sans se prononcer sur son opportunité - ou comme celle (proposée par le doyen Vedel) dite du « lit de justice » du pouvoir constituant. Or, il est évident que procéder à un contrôle de constitutionnalité des lois de révision anéantit une telle justification, car la cour se trouve alors incontestablement dotée du dernier mot sur le fond des réformes. Sans doute l'abandon d'une justification aussi puissante apparaît-il trop coûteux à beaucoup de juridictions constitutionnelles.

Pourtant, la Cour suprême indienne ne va pas s'en tenir à cette prudence initiale.

«La doctrine indienne de la structure basique de la Constitution. Un socle indérogeable et flexible? », Les Cahiers du Conseil constitutionnel, $\mathrm{n}^{\circ} 27,2009$, p. 76-77. 


\section{Deuxième phase : La menace du contrôle}

Le changement d'attitude de la Cour suprême a en fait été consécutif aux réactions des parlementaires devant son activisme juridictionnel. En effet, se heurtant au contrôle sourcilleux de la Cour sur ses réformes législatives, notamment en matière de protection du droit de propriété, le Parlement va s'efforcer de surmonter la jurisprudence de cette dernière en révisant la Constitution sur ce point précisément - il s'agit du XVII ${ }^{\mathrm{e}}$ amendement. Après une première décision, par laquelle les juges avaient épargné cet amendement ${ }^{1}$, la Cour opère un revirement de jurisprudence en 1967, dans l'arrêt fondateur Golak Nath v. State of Punjab ${ }^{2}$. Elle juge en effet qu'un amendement constitutionnel ne doit pas pouvoir porter atteinte à un droit fondamental, en vertu de la garantie que la Constitution leur accorde contre «toute loi »-même si la Cour ne pose ce principe que pour l'avenir et n'annule donc pas l'amendement contestés.

Dès 1967, donc, la Cour suprême indienne a adopté la position qui est aujourd'hui celle des cours allemande ou italienne, par exemple : celles-ci en effet procèdent au contrôle des révisions constitutionnelles, mais (jusqu'à aujourd'hui en tout cas) ne sont jamais allées jusqu'à en invalider une : sans doute la légitimité dont les amendements constitutionnels sont dotés (du fait de la procédure permettant leur adoption) constitue-t-elle un obstacle qui mérite considération... Mais pourquoi alors se reconnaître compétentes pour les examiner - dans la mesure où cela semble ruiner la justification issue de la théorie de l'aiguilleur, sans que les cours en tirent apparemment l'avantage escompté (puisqu'elles se refusent à invalider de telles lois) ? En réalité, le bénéfice que les juges peuvent retirer d'une telle position n'est pas négligeable - car il en va du jeu institutionnel comme de celui des échecs : la persistance de la menace est souvent plus efficace encore que son exécution. En contrôlant les lois de révision sans jamais les déclarer inconstitutionnelles, les cours allemande et italienne les enserrent dans un «maillage » de principes jurisprudentiels de plus en plus fin - ce qui conduit les autorités politiques à se montrer fort prudentes lorsqu'elles entendent procéder à des modifications du texte constitutionnel. Le développement d'une telle jurisprudence leur permet également de justifier le recours à la technique dite de «l'interprétation conforme » vis-à-vis des lois constitutionnelles. Bref, un tel contrôle, qui reste " platonique », peut se révéler à la fois indolore pour les autorités politiques (qui ne voient pas leurs révisions invalidées) et très bénéfique (en termes d'exercice du pouvoir) pour les cours, puisqu'elles deviennent des acteurs essentiels du processus constituant. Il faut simplement noter qu'en adoptant une telle position, les cours donnent à voir de façon beaucoup plus nette leur pouvoir politique - ce qui tend à les exposer à certaines critiques. Cela n'a pas empêché la Cour suprême d'aller plus loin encore.

1. Sajjan Singh v. State of Rajasthan, A.I.R. 1965 SC 845.

2. Golak Nath v. State of Punjab, A.I.R. 1967 SC 1643.

3. Il s'agit d'un prospective overruling, pour reprendre l'expression consacrée - c'est-àdire un revirement pour l'avenir, qui n'affecte pas la validité des amendements contrôlés en l'espèce. 


\section{Troisième phase : La mise en ouvre du contrôle}

En 1973, dans un arrêt Kesavananda Bharati v. State of Kerala ${ }^{1}$, la Cour va tout simplement déclarer inconstitutionnel un amendement à la Constitution. Comment comprendre, eu égard à la légitimité qui entoure normalement de tels actes, cette audace de la Cour - une audace qui, même si elle tend à se répandre ${ }^{2}$, reste tout de même assez rare dans le monde ? La réponse doit sans doute être recherchée dans la spécificité de la situation indienne, en particulier durant la période la plus conflictuelle entre les parlementaires et les juges.

D’une part, le consensus politique qui est supposé présider à toute révision constitutionnelle n'était guère réalisé à ce moment-là : le Parti du Congrès a en effet disposé, jusqu'à la fin des années 1970, d'une majorité telle au Parlement qu'il était en mesure de procéder, seul, à certaines réformes constitutionnelles. Pour la plupart des matières en effet, la majorité qualifiée exigée (par l'art. 368) pour la révision du texte de la Constitution n'est pas très forte :il suffit d'un vote aux deux tiers des voix dans chacune des chambres pour qu'elle soit adoptée $e^{3}$. Dès lors, un seul parti (aussi puissant et populaire soit-il) étant en mesure d'amender la Constitution, la Cour a pu se présenter comme la protectrice des droits de la minorité - puisque la procédure de révision permettait de se passer du consentement de cette dernière.

D'autre part, et surtout, cette situation politique originale avait donné un tour particulier à l'affrontement entre le Parlement et la Cour suprême. La tentation a en effet été grande, pour le Parti du Congrès, de recourir à la procédure de révision de la Constitution pour surmonter à sa guise les décisions de la Cour invalidant ses lois ordinaires. Aussi, la seule ressource dont ait disposé la Cour pour conférer à sa jurisprudence une certaine efficacité a été la menace d'invalider également les amendements par lesquels la majorité parlementaire tentait de la contourner : tel était le sens de l'arrêt Golak Nath de 1967, par lequel elle interdisait dorénavant au Parlement de porter atteinte, par voie de révision, aux droits fondamentaux reconnus dans la $\mathrm{III}^{\mathrm{e}}$ Partie de la Constitution (celle sur laquelle elle s'appuyait essentiellement pour s'opposer à la politique gouvernementale).

Or, précisément, le Parlement a réagi très vite à cette décision en adoptant, en 1971, sous l'impulsion d'Indira Gandhi, une révision de la Constitution (le $24^{\mathrm{e}}$ amendement), qui affirmait le caractère souverain du pouvoir de révision, non seulement en l'indiquant expressément dans l'article 368 lui-même (al. 1 et 3 , précités), mais aussi en ajoutant à l'article 13 (précité) un 4 ealinéa, selon lequel : «Rien dans le présent article ne saurait viser les amendements adoptés selon la procédure de l'art. 368 ».

L'arrêt Kesavananda ne constitue donc que la riposte de la Cour à cette tentative des autorités gouvernementales de neutraliser la menace qu'elle avait

1. Kesavananda Bharati v. State of Kerala, A.I.R. 1973 SC 1461.

2. Ainsi que l'attestent notamment les exemples du Bénin en 2006 et de la Turquie en 2008 (supra, notes 7 et 8 ).

3. Pour les autres matières (énumérées à l'art. 368, al. 2), il suffit qu'à ce vote parlementaire succède une ratification du texte par les législatures de la moitié des États. 
proférée dans Golak Nath : tout en admettant la validité du $24^{\mathrm{e}}$ amendement (et donc la possibilité pour le Parlement de réviser n'importe quelle partie de la Constitution - y compris celle consacrant les droits fondamentaux), elle y déclare inconstitutionnelle une partie du $25^{\mathrm{e}}$ amendement (qui excluait tout recours contre la fixation par le Parlement du montant de la compensation pour expropriation). Le motif invoqué par la Cour est que les amendements à la Constitution, s'ils peuvent toucher à n'importe quelle partie du texte, ne sauraient néanmoins recevoir n'importe quel contenu : ils ne doivent notamment pas porter atteinte à la «structure basique de la Constitution» (sans que le sens de cette expression soit précisé). Dans plusieurs décisions (par exemple dans Indira Nehru Gandhi v. Raj Narain de 1975¹), la Cour a par la suite eu l'occasion d'utiliser cette jurisprudence restrictive du pouvoir de révision. Elle franchira pourtant une étape supplémentaire, afin de prémunir son contrôle contre toute tentative de résistance de la part du Parlement.

\section{Quatrième phase : La garantie du contrôle}

La Cour suprême indienne, poussée dans ses retranchements par le Parlement, a en effet été amenée à adopter une solution tout à fait inédite, dans le cadre de son contrôle des amendements constitutionnels. En effet, devant l'opiniâtreté des juges, les parlementaires ont décidé de riposter en adoptant un nouvel amendement à la Constitution en $1976\left(\mathrm{le} 42^{\mathrm{e}}\right)$ : ils ont ajouté à l'article 368 deux alinéas qui, d'une part, interdisaient de remettre en cause (« devant quelque cour que ce soit, et sur quelque fondement que ce soit») une loi de révision constitutionnelle et, d'autre part, (« pour ôter tout doute » à ce sujet) réaffirmaient le caractère illimité du pouvoir de révision constitutionnelle (al. 4 et 5 de l'art. 368). La Cour n'a pas flanché pour autant : dans une décision Minerva Mills Ltd and others v. Union of India and Others de 1980, elle a même jugé que ces deux alinéas précisément étaient inconstitutionnels, dans la mesure où ils portaient atteinte à la fameuse «structure basique de la Constitution ». L'ultime riposte de la Cour a donc consisté à annuler les amendements mêmes par lesquels le Parlement cherchait à lui dénier le droit de les contrôler.

On a donc assisté, pendant une quinzaine d'années, à une véritable guerre de tranchées - plutôt qu'à une simple bataille ${ }^{2}$ - entre la Cour suprême et le Parlement indiens. Notons que si le conflit s'est petit à petit estompé à partir de la décision Minerva Mills, cela résulte moins, semble-t-il, d'une capitulation du Parlement devant la puissance de la Cour, que d'une recomposition politique (de part et d'autre) rendant moins brutale l'opposition idéologique entre les parlementaires et les juges. Ainsi, le conflit ne cesse pas au seul motif que la Cour suprême procède à l'annulation de lois de révision. En effet, le Parlement

1. Indira Nehru Gandhi v. Raj Narain, A.I.R.1975 SC 2299.

2. Edward McWhinney, op. cit., p. 134. Selon la formule de Granville Austin (Working a Democratic Constitution: The Indian Experience, Oxford University Press India, 1999, p. 198) : « a great war (...) over parliamentary versus judicial supremacy ». 
et son gouvernement n'ont pas comme seule ressource la modification de la Constitution, pour faire échec à la politique jurisprudentielle de la Cour : ils peuvent également tenter de jouer sur son organisation ou sa composition même - ce qui ne nécessite pas toujours un amendement

constitutionnel.

Indira Gandhi ne s'en du reste pas privée : en 1973 et 1976 par exemple, elle a décidé de ne pas se conformer aux règles conventionnelles d'avancement à l'ancienneté pour l'accession au poste de Chief Justice - afin d'y placer des personnalités plus sensibles aux positions gouvernementales ${ }^{1}$. Pour le dire autrement, le jeu (des rapports de force) ne s'arrête jamais ${ }^{2} \ldots$

Ainsi la théorie des contraintes permet-elle d'expliquer, autrement qu'en invoquant les progrès de l'État de droit, les prises de position des cours constitutionnelles quant à la justiciabilité des lois de révision : celles-ci se comprennent alors comme le résultat d'un jeu contingent de rapports de forces entre organes constitutionnels - un jeu dont les cours, loin d'être les arbitres désintéressés, font résolument partie.

\section{LES CONTRAINTES DE LA JUSTIFICATION DU CONTRôLE DES LOIS CONSTITUTIONNELLES}

Les arguments de la Cour suprême ne sont pas toujours faciles à analyser, tant les opinions dissidentes ou concurrentes sont riches et complexes : la décision fondamentale Kesavananda Bharati v. State of Kerala (que l'on a pu qualifier de plus importante décision constitutionnelle ${ }^{3}$ en Inde) comporte par exemple huit cents pages et de multiples opinions croisées des juges constitutionnels, sur chaque point de l'argumentation - de sorte qu'il est bien difficile de la résumer.

1. Edward McWhinney, op. cit., p. 138.

2. À cet égard, il convient également de souligner qu'au moment même où, en tant que chef du gouvernement, elle a décidé de proclamer l'état d'urgence, le 26 juin 1975 - avec tous les moyens que ce régime d'exception lui autorisait en termes de restrictions aux libertés - Indira Gandhi a également fait insérer dans la Constitution une disposition interdisant à la justice de se saisir de la question de la régularité de l'élection du Premier Ministre, précisément parce qu'un juge l'avait accusée de fraude électorale (il s'agit du $39^{\mathrm{e}}$ amendement à la Constitution, datant de 1975). La Cour suprême a alors déclaré cet amendement inconstitutionnel sur le fondement de la théorie de la «structure basique de la Constitution » : il lui aurait sans doute été difficile de faire autrement sans se discréditer gravement, tant ledit amendement était outrancier. Mais la Cour n’a pas pour autant osé, en ces circonstances très tendues, invalider l'élection d'Indira Gandhi (il s'agit de l'arrêt Indira Nehru Gandhi v. Raj Narain, AIR 1975 SC 2299). Aussi peut-on maintenir, plus généralement, que la conscience, chez les juges, des instruments dont disposent les autorités politiques pour les atteindre (nominations, augmentation du nombre de juges, mise en accusation, etc.) suffit le plus souvent à les convaincre de faire un usage prudent de la théorie de la «structure basique ».

3. Gary Jeffrey Jacobsohn : «An unconstitutional constitution? A comparative perspective », I-CON, Vol. 4, $\mathrm{n}^{\circ} 3$ (juillet 2006). 
En même temps, la richesse des justifications se révèle fort instructive pour qui souhaite reconstituer les débats théoriques ayant traversé la Cour dans chacune de ses décisions. Il ne sera cependant ici question que de dresser un tableau très (trop) schématique de l'évolution des justifications avancées par la Cour quant à son contrôle de la régularité des amendements constitutionnels. Cette analyse succincte permettra cependant d'identifier un tournant majeur dans la manière dont les juges se sont justifiés, tournant qu'il conviendra dans un second temps d'expliquer en termes de contraintes de l'argumentation.

\section{Le tournant argumentatif}

L'acte fondateur en la matière intervient avec la décision Golak Nath v. State of Punjab : par une courte majorité de 6 contre 5, le principe est en effet posé selon lequel désormais les droits fondamentaux seraient placés hors d'atteinte du pouvoir d'amendement. L'argumentation de la Cour peut être résumée comme suit : le pouvoir d'amendement de l'art. 368 étant créé par la Constitution, il ne peut s'exercer que dans le cadre que celle-ci lui assigne ; or son article 13 déclare nulle toute loi portant atteinte à l'un des droits consacrés dans la III ${ }^{\mathrm{e}}$ Partie, et un amendement ne saurait être analysé que comme une sorte de loi ; dès lors, le Parlement n'a pas le droit, par un amendement adopté selon la procédure de l'article 368, de porter atteinte à l'un des droits fondamentaux consacrés dans la Constitution.

Dans cette décision, la Cour formule donc à la fois une théorie du pouvoir de révision comme étant par nature limité par la Constitution (à travers notamment une analyse des termes de l'article 368), et une théorie des limites rencontrées par ce pouvoir de révision : celles-ci sont constituées par les droits fondamentaux consacrés dans la $\mathrm{III}^{\mathrm{e}}$ Partie, auquel le Parlement ne saurait porter atteinte (pas plus en tant que constituant dérivé qu'en tant que simple législateur) - l'article 13 étant invoqué comme un argument textuel pour justifier cette interdiction.

Comme on l'a vu, la majorité parlementaire va précisément contrer cette argumentation en 1971, par une révision constitutionnelle qui d'une part indique explicitement que les amendements constitutionnels ne font pas partie des « lois » mentionnées à l'article 13 (devant donc être déclarées nulles en cas d'atteinte à un droit fondamental) et qui d'autre part affirme tout aussi nettement que le pouvoir de révision accordé au Parlement par l'article 368 de la Constitution peut concerner toutes les parties de la Constitution (y compris donc la III ${ }^{\mathrm{e}}$ Partie).

La Cour suprême n'a pas cherché à invalider ce $24^{\mathrm{e}}$ amendement - et a même confirmé sa conformité à la Constitution, dans le fameux arrêt Kesavananda Bharati v. State of Kerala de 1973․ Ce faisant, elle a néanmoins réaffirmé son intention de procéder au contrôle de la régularité des révisions constitutionnelles - tout en modifiant sensiblement sa justification à cet égard. C'est en effet

\section{Supra.}


à l'occasion de cette décision qu'elle a introduit la doctrine de la «structure basique de la Constitution » : les juges ont estimé ${ }^{1}$ que l'article 368 n'habilitait pas le Parlement à altérer cette «structure basique», au motif que le pouvoir d'amender ne saurait être compris comme un pouvoir de détruire. Estimant qu'une partie du $25^{\mathrm{e}}$ amendement (excluant la compétence des cours en certaines matières ${ }^{2}$ ) portait précisément atteinte à cette «structure basique », la Cour l'a jugée irrégulière.

Cette théorie justificative a depuis lors été consacrée par la jurisprudence et a servi à annuler plusieurs amendements constitutionnels jusqu'à aujourd'hui ${ }^{3}$ - même si il n'y a bien sûr jusqu'ici aucun accord sur le contenu de cette « structure basique », la Cour s'étant sagement abstenue d'adopter une position nette sur le sujet ${ }^{4}$.

On observe donc que la Cour suprême indienne est passée d'une justification s'appuyant sur le texte même de la Constitution (notamment sur l'article 13, mais aussi sur l'article 368) à une justification fondée sur l'idée d'un socle de valeurs, règles ou principes auxquels le pouvoir de révision, en raison de sa nature limitée, ne pourrait pas toucher.

Or, il est tout à fait frappant de constater que l'on retrouve une telle justification - quoique sous des formulations différentes, bien sûr - chez les autres juridictions constitutionnelles qui se sont reconnues compétentes pour contrôler la régularité des lois de révision. Toutes invoquent l'idée d'un ensemble de principes ou de valeurs censé être au fondement, ou à la base de la constitution considérée comme un tout.

Tel est le cas en Italie par exemple : la Cour, à partir de 1988, a jugé irrégulière la révision des principes qui « appartiennent à l'essence des valeurs suprêmes sur lesquelles se fonde la Constitution italienne $»^{5}$. Une idée assez semblable

1. Du moins, cette thèse a-t-elle été acceptée par une majorité de 7 juges (Sikri, Shelat, Hedge, Grover, Jaganmohan Reddy, Khanna et Mukherjea) contre 6. La minorité a au contraire soutenu que le Parlement devait rester souverain dans l'exercice de son pouvoir de révision constitutionnelle : cela a été le cas des juges Ray, Palekar, Beg, Dwivedi, Mathew et Chandrachud (cf.V. N. Shukla : Constitution of India, Delhi, Eastern Book Company, $8^{e}$ éd. by M. P. Singh, 1990, p. 709).

2. Cf. supra.

3. Ainsi notamment dans les arrêts Maharao Sahib Shri Bhim Singhji v. Union of India de 1981 et IR Coelho (Dead) by RLRS v. State of Tamil Nadu de 2007.

4. Dans Kesavananda Bharati, les juges étaient déjà en désaccord sur la consistance de la notion - et c'est ce qui explique que le $24^{\mathrm{e}}$ amendement (qui levait l'interdiction, posée par l'arrêt Golak Nath, d'amender la III' Partie de la Constitution relative aux droits fondamentaux) n'ait pas été invalidé. En effet, l'un des 7 juges ayant adopté la doctrine de la «structure basique » (le juge Khanna) avait estimé que les droits fondamentaux ne faisaient pas partie de cette structure basique, faisant basculer la majorité du côté de ceux qui souhaitaient confirmer la validité de l'amendement.

5. Cour Constitutionnelle italienne, arrêt n ${ }^{\circ} 1146$ de 1988. La Cour estime en effet, dans cette décision, que ces principes suprêmes « consistent tant dans les principes que la Constitution prévoit comme limites absolues au pouvoir de révision constitutionnelle, telle la forme républicaine (art. 139 de la Constitution), que dans les principes qui, bien 
est avancée par la Cour constitutionnelle turque qui, dans sa décision du 6 juin 2008, a annulé un amendement constitutionnel portant atteinte à " l'ordre constitutionnel » (lequel comprend non seulement les trois premiers articles de la Constitution, ainsi que le quatrième qui met ces derniers à l'abri de toute révision, mais aussi l'ensemble des normes constitutionnelles qui concrétisent cet ensemble ${ }^{1}$ ). De la même façon, la Cour constitutionnelle du Bénin indique, pour justifier son annulation d'une révision constitutionnelle, qu'un tel acte doit tenir « compte des idéaux qui ont présidé à l'adoption de la Constitution $»^{2}$. Plus récemment encore, la Cour constitutionnelle de Karlsruhe, qui jusque-là procédait à une interprétation plutôt stricte du fameux article 79 al. 3 de la Loi fondamentale (rendant « intangibles » certaines dispositions ou principes constitutionnels), s'est montrée plus audacieuse dans la décision du 30 juin 2009, relative au Traité de Lisbonne $^{3}$ : elle prétend déduire de l'article 79 al. 3, l'existence d'un noyau dur de l'«identité constitutionnelle » allemande, à laquelle le pouvoir de révision constitutionnelle lui-même ne pourrait porter atteinte (en l'occurrence pour aller plus loin sur le chemin de l'intégration européenne). Dans le contexte il est vrai un peu particulier de la construction européenne, la Cour a donc substitué le « contrôle du respect de l'identité constitutionnelle » à celui du respect de l'art. 79, al. 3 même si celui-ci est conçu comme découlant de celui-là ${ }^{4}$.

Une telle communauté de vues, réunissant des juges aux cultures constitutionnelles aussi diverses, ne peut être purement fortuite. Si l'on ne cède pas à la tentation d'y voir l'effet d'une sorte de révélation qui aurait touché successivement ces différentes cours, il faut la comprendre comme le résultat des contraintes qui pèsent sur le raisonnement de celles-ci.

\section{Les contraintes de l'argumentation}

Ces contraintes peuvent en réalité être de plusieurs ordres.

La relative uniformité signalée à l'instant tient d'abord à la structure de l'argumentation juridictionnelle. Michel Troper a parfaitement montré que cette dernière conduisait les juges, dans leur contrôle des lois ordinaires, à produire

que n'étant pas mentionnés expressément parmi ceux qui ne peuvent pas être soumis à la procédure de révision constitutionnelle, relèvent de l'essence même des valeurs suprêmes sur lesquelles se fonde la Constitution italienne ».

1. Voir sur le sujet Ibrahim Ö. Kaboglu : «Le contrôle juridictionnel des amendements constitutionnels en Turquie », Cahiers du Conseil constitutionnel, $\mathrm{n}^{\circ} 27-2009$, p. 38 et s.

2. Cour constitutionnelle du Bénin, arrêt du 8 juillet 2006. V. Babakane D. Coulibaley : «La neutralisation du Parlement constituant (à propos de la décision n ${ }^{\circ} \mathrm{DCC}$ 06-074 du 8 juillet 2006 de la Cour constitutionnelle du Bénin)», op. cit., not. p. 1510.

3. Cour constitutionnelle fédérale, arrêt 2 BvE 2/08 et autres, arrêt du 30 juin 2009, Traité de Lisbonne.

4. Sur ce sujet, on peut se reporter à la remarquable étude d'Oliver Lepsius : «Le contrôle par la Cour constitutionnelle des lois de révision constitutionnelle dans la République fédérale d'Allemagne », Cahiers du Conseil constitutionnel, n²7-2009, p. 13 et s. (notamment pp. 20-21). 
des principes « généraux » permettant l'interprétation des dispositions constitutionnelles ${ }^{1}$ - et qu'une fois ces principes construits, il pouvait être tentant pour eux («pourvu que l'occasion se présente ») d'en faire des principes « supérieurs » s'imposant au pouvoir de révision constitutionnelle ${ }^{2}$. C'est ce qui lui permettait de conclure, sous la forme (coutumière chez lui) d'un renversement causal tout à fait éloquent, que « ce n'est pas l'existence de principes qui conduit au contrôle, c'est au contraire le contrôle qui conduit à la création de principes » ${ }^{3}$.

Reste qu'il existe plusieurs façons d'envisager ces principes «supérieurs », et que l'on doit se demander pourquoi ils le sont, globalement, de façon semblable. On aurait en effet pu penser, au contraire, que les cours pouvant s'appuyer sur des dispositions constitutionnelles qui interdisent formellement la révision de certains articles se contenteraient de celles-ci, en invoquant la lettre du texte : cela paraît en effet suffire à justifier leur contrôle des amendements constitutionnels, tout en s'en tenant strictement à leur rôle de "gardiens de la Constitution $»^{4}$.

Une première explication, apparemment simple, doit être écartée. Elle consiste à suggérer que l'invocation de la «structure basique de la constitution » (ou de toute autre théorie semblable) permettrait la mobilisation d'un plus grand nombre de principes que le simple appel au texte constitutionnel - de sorte que cette théorie lui ouvrirait un champ plus large de normes de référence pour son contrôle de la régularité des amendements. Cette idée est en réalité très contestable : il convient de ne pas négliger la liberté du juge dans l'interprétation des articles de la constitution. Si le Conseil constitutionnel s'aventurait un jour à contrôler les lois de révision, par exemple, on peut imaginer qu'en s'appuyant sur le seul art. 89 al. 5 , il pourrait invoquer à peu près n'importe quel principe fondamental. Il lui suffirait ainsi de définir la « forme républicaine du gouvernement » comme une référence implicite aux « valeurs qui fondent notre Constitution », pour lui faire jouer exactement le même rôle que la théorie de

1. Michel Troper indique ainsi que pour justifier une interprétation «il faut nécessairement faire appel à un principe d'interprétation ou à une norme plus générale que le texte interprété. Un acte administratif s'interprète "à la lumière" de la loi qu'il exécute, une loi "à la lumière" de la constitution, la constitution elle-même à la lumière d'un principe encore plus général » («La notion de principes supra-constitutionnels », in La théorie du droit, le droit, l'État, Paris, PUF, “Léviathan”, 2001, p. 212).

2. Il écrit ainsi que «si l'on entend contrôler les lois constitutionnelles, elles doivent être conformes (...) à certaines règles de fond. On peut alors invoquer certaines dispositions générales de la constitution même ou l'“esprit” de la constitution, c'est-à-dire des principes obtenus par généralisation de l'ensemble des normes constitutionnelles en vigueur, (et) on ne peut éviter (...) de leur conférer une valeur supérieure à celle des lois constitutionnelles » (ibid., p. 213).

3. Ibid.

4. Et de fait, la Cour constitutionnelle de Karlsruhe, par exemple, s'est jusqu'à la décision de 2009 contentée d'invoquer l'art. 79 al.3 pour justifier sa compétence à l'égard des lois de révision. On doit du reste noter que même la Cour suprême indienne a initialement tenté de fonder son contrôle sur un argument de texte - en l'occurrence l'article 13 protégeant les droits fondamentaux (dans Golak Nath). 
la «structure basique ». De la même façon, il est probable que la Cour suprême indienne aurait pu justifier n'importe quelle limitation du pouvoir d'amendement en s'appuyant sur les seuls droits fondamentaux de la III ${ }^{e}$ Partie de la Constitution de 1950. En réalité, la supériorité de la théorie de la structure basique sur les arguments de texte tient moins à l'amplitude supposée de son contenu, qu'à sa forme même.

En effet, les arguments de texte sont affectés d'une faiblesse congénitale, que l'on peut exprimer très platement : un texte, cela se modifie. Dès lors, si une cour empêche les autorités politiques de modifier certaines dispositions de la constitution en se fondant sur une ou plusieurs (autres) dispositions de la constitution, rien ne garantit que ces dernières ne soient pas un jour ellesmêmes supprimées ou modifiées par un amendement. Or, prétendre interdire aussi cette dernière révision suppose nécessairement de se référer à des principes "supérieurs". Cependant, l'existence de tels principes ne peut se justifier que de deux façons. La première consiste à les trouver en dehors du texte, et donc à invoquer un prétendu «droit naturel », qui s'imposerait à la constitution elle-même ; mais les juges sont peu enclins, en général, à admettre que leur tâche consiste à faire respecter autre chose que la constitution - la référence au droit naturel étant d'un maniement délicat, dans des sociétés qui se présentent comme démocratiques. La seconde (qui semble dès lors la plus raisonnable) réside bien sûr dans l'invocation d'une supposée « structure basique de la constitution » (quelle que soit la manière dont on la désigne) que le juge prétend tirer de son texte même.

Le cas indien est à cet égard significatif : dans Golak Nath, la Cour suprême s'était appuyée sur l'article 13 (surtout), mais aussi sur la rédaction de l'article 368, pour invalider un amendement ; et c'est lorsque le Parlement a modifié la rédaction de ces deux articles afin de lui interdire le contrôle des lois de révision, que la Cour a été conduite à modifier radicalement sa justification, et à imaginer la théorie de la «structure basique ».

On comprend mieux, dès lors, le succès grandissant de cette dernière : si l'on se place du point de vue d'une cour qui désire contrôler la validité des amendements constitutionnels, cette théorie apparaît bien comme la seule à même de garantir que les dispositions constitutionnelles de son choix (y compris celles par lesquelles elle justifie cette protection ${ }^{1}$ ) ne pourront être modifiées ${ }^{2}$ - sans pour autant sortir trop ouvertement de son rôle d'interprète (soumis)

1. Rien n'empêche en effet la cour d'invoquer à cet égard des dispositions textuelles contenues dans la constitution, les deux types de justifications n'étant nullement exclusives l'une de l'autre : les cours constitutionnelles les utilisent du reste toujours toutes les deux.

2. Une révision qui prétendrait altérer les compétences de la cour, d'une manière ou d'une autre, pourrait évidemment être invalidée comme portant atteinte à la «structure basique » de la constitution (telle a du reste été la réaction de la Cour suprême indienne) - si bien que l'adoption de cette théorie apparaît comme une façon pour les cours de se prémunir contre d'éventuelles mesures de rétorsion de la part d'autorités politiques mal disposées à leur égard. 
de la Constitution. On observe du reste que, quelle que soit la formulation de cette thèse, elle revient toujours à "dégager » de l'ensemble des dispositions constitutionnelles une série de principes (qui peuvent être consacrés ou non dans le texte), par un procédé qui se présente comme inductif. D’un côté, par conséquent, la Cour semble se conformer à son travail habituel en procédant à de simples interprétations du texte constitutionnel - sans paraître s'adonner à de vaines spéculations. De l'autre cependant, il est évident que ces principes ne se réduisent jamais aux dispositions constitutionnelles qui les énoncent : ces dernières se présentent au mieux comme la concrétisation desdits principes, puisqu'ils sont censés exister indépendamment de ces dispositions - lesquelles ne sont finalement, du point de vue des juges, que la manifestation, le signe de cette existence ${ }^{1}$.

$\mathrm{Au}$ terme de cette analyse, on comprend donc, pour le dire aussi simplement que possible, que les cours constitutionnelles ne puissent pas s'appuyer sur le seul texte constitutionnel pour protéger de façon efficace le texte constitutionnel : une telle tentative serait aussi vaine que celle du Baron de Münchausen, qui prétendait s'être extirpé d'un marécage en se tirant par ses propres cheveux. Mais n'est-ce pas là simplement une autre manière de relever le caractère éminemment paradoxal de l'ambition que nourrissent nos constitutions de pouvoir déterminer les conditions de leur propre création?

1. Cela est précisément ce qui permet de justifier que l'on puisse interdire à un amendement de supprimer une disposition qui limite le pouvoir de révision. 